\title{
Acuity for orientation measured with a sequential recognition task and signal detection methods
}

\author{
ETHEL MATIN and ANNA DRIVAS \\ C. W. Post Center, Long Island University, Greenvale, New York 11548
}

\begin{abstract}
An orientation recognition task with two stimuli was employed as the basis of a psychophysical method for measuring sensitivity to orientation. Essentially, this method depends on a subject's ability to discriminate between two stimuli differing in orientation and presented to the same retinal area in random succession from trial to trial. Advantages of this sequential method over traditional simultaneous matching methods are discussed. Its feasibility is demonstrated in a signal detection analysis of acuity for the orientation of short luminous slits presented foveally on a dark field at seven reference orientations varying between $0^{\circ}$ and $90^{\circ}$. In both subjects employed, sensitivity was greater for horizontal and vertical orientations than for other slopes (the oblique effect).
\end{abstract}

Stimulated by the rapidly developing neurophysiological literature on cortical cells sensitive to the orientations of lines, bars, edges, and gratings, there has occurred in the past decade a quickened interest in the psychophysics of perceived tilt. This interest is manifest in studies which measure the variability of judgments of perceived slope, i.e., in studies of the sensitivity or acuity of the ability to discriminate orientation (Andrews, 1965, 1967a, 1967b; Bouma \& Andriessen, 1968). It can also be seen in the large number of recent studies devoted to the various tilt illusions and aftereffects in which measures of the average perceived orientation are of primary concern (Blakemore, Carpenter, \& Georgeson, 1970; Bouma \& Andriessen, 1970; Campbell \& Maffei, 1971; Carpenter \& Blakemore, 1973; Georgeson, 1976; Lennie, 1971; Matin, 1974; Mitchell \& Muir, 1976; Muir \& Over, 1970; O'Toole \& Wenderoth, 1977; Over, Broerse, \& Crassini, 1972; Oyama, 1975; Thomas \& Shimamura, 1975; Tolhurst \& Thompson, 1975; Wallace, 1975; Wallace \& Crampin, 1969; Wenderoth \& Curthoys, 1974).

Despite this great current interest in the experimental psychology of perceived tilt, relatively little effort has been devoted to developing psychophysical techniques that permit a full exploitation of the exciting physiological discoveries that stimulated the

\footnotetext{
This report was supported by BNS-76-15298 from the National Science Foundation and by an award from the C. W. Post Center Research Committee. It is based on a thesis, entitled "A signal detection analysis of the oblique effect measured with an orientation recognition task," presented by Anna Drivas to the faculty of the Graduate Studies Program, C. W. Post College of Long Island University, in partial fulfillment of the requirements for the degree of Master of Arts, June 1978. Requests for reprints should be sent to Ethel Matin, Department of Psychology, C. W. Post College, Long Island University, Greenvale, New York 11548.
}

interest. This is true of both the methods for measuring average perceived orientation and the techniques for measuring sensitivity. Some inadequacies of the former have been considered in recent papers by Fisher (1973) and by Wenderoth and his co-workers (Emerson, Wenderoth, Curthoys, \& Edmonds, 1975; Wenderoth, Beh, \& White, 1978). The relatively neglected measurement of sensitivity is of primary concern in the present report.

\section{Traditional Techniques for Measuring Sensitivity}

Psychophysical studies of acuity for tilt date back to the last century (Jastrow, 1893) and fall roughly into two categories: experiments employing absolute judgment methods and experiments employing matching techniques. With the former, the subject makes verbal reports on perceived orientation. The standard deviations of these reports is used to measure sensitivity (Fisher, 1969, 1974; Keene, 1963; Smith, 1962; Alluisi, Note 1). With the matching techniques, subjects are required to compare two contours present simultaneously in the visual field. In some studies, this match involves the orientations of two lines (the parallel alignment method employed by Andrews, 1967a, 1967b; Rochlin, 1955). In others, a dot is set to the apparent extension of the line for which orientation is being assessed (the dot alignment technique, employed by Bouma \& Andriessen, 1968, and Salomon, 1947). With this method, the matching line is essentially represented by its end points. A third matching method employed in studies of perceived tilt involves a comparison of two angles (Lennie, 1971; Emerson et al., 1975). To our knowledge, however, this latter method has been employed only in studies of average perceived tilt, not in studies primarily concerned with sensitivity. Any of the matching methods can be employed in conjunc- 
tion with any of the conventional psychophysical techniques such as constant stimuli, limits, adjustment, etc. Some measure of judgmental variability (such as the standard deviation of a subject's settings in the method of adjustment) is used to index sensitivity.

A year ago, we undertook an assessment of these various techniques with a view toward selecting a method suitable for a systematic program of research on sensitivity to orientation. It was immediately apparent that the absolute judgment methods were too coarse for this purpose. The matching methods were also unsatisfactory, primarily because they do not lend themselves to studies with gratings. This is particularly obvious with the dot alignment and angle matching techniques; it also applies, however, to the parallel alignment method. Consider, for example, the problems that arise if an attempt is made to measure acuity for the orientation of gratings of various spatial frequencies by using a matching line. The Fourier spectrum of the line would somehow have to be taken into account, as would the retinal position of the line and the grating. It is not at all obvious that this could be done in a satisfactory way; in any event, a variety of control experiments would be required. In addition, when gratings of low spatial frequency are used, the visual field is simply not large enough to contain simultaneously both the line and a grating with enough cycles to warrant use of the term.

Even when the matching methods are used only for the simpler problem of measuring acuity for the orientation of a single test line, control experiments are required to determine the influence of the matching stimulus on the judgment. With the dot alignment method, for example, the effect of retinal position of the dot and of its distance from the test line must be determined. In the parallel alignment method, the length of the matching line, its retinal position, and its distance from the test line are among the variables that must be considered (see Emerson et al., 1975; O'Toole \& Wenderoth, 1977; and Wenderoth, Bey, \& White, 1978, for experimental evidence on these questions). In essence, for both gratings and lines, the matching methods measure the variance of the difference between the perceived tilt of the test stimulus and the matching stimulus; no satisfactory method is available for separating the components of this distribution of differences.

\section{An Alternative Technique}

The inadequacies of traditional methods for measuring acuity for slope motivated development of the new technique that is the subject of this report. Essentially, this technique is based on a subject's ability to discriminate between two stimuli differing in orientation and presented in random succession to the same retinal area rather than simultaneously to different retinal areas (as is the case with the matching methods). The subject's judgmental task on each trial involves orientation recognition rather than the conventional simultaneous pair comparison. We used this method to measure sensitivity to the orientations of short luminous slits on a dark field at seven reference orientations: $0^{\circ}, 15^{\circ}, 30^{\circ}, 45^{\circ}, 75^{\circ}$, and $90^{\circ}$. Details of the procedure and of techniques for processing the data will be described in the sections on methods and on results. For the moment, we will simply note that our two observers were able to make sensitive and reliable discriminations with this judgmental task. The data, moreover, had the important advantage that they lent themselves easily to processing with standard signal detection methods. As might be expected from the literature on the "oblique effect," sensitivity in both observers was greater at the horizontal and vertical reference orientations than at the obliques.

\section{METHOD}

\section{Subjects}

One of the authors (E.M.) and a graduate student of experimental psychology (M.V.) served as subjects. The former was familiar with the procedure and was an experienced observer, while the latter was naive about the experiment and had not previously participated in a psychophysical study. Subject E.M. wore glasses to correct for a myopia of $-.5 \mathrm{D}$ and an astigmatism of $-.5 \mathrm{D}$, axis $180^{\circ}$ in her right (viewing) eye. Subject M.V.'s contact lenses corrected a myopia of $-5.25 \mathrm{D}$ and an astigmatism of $+.25 \mathrm{D}$, axis $80^{\circ}$ in her right eye.

\section{Apparatus}

The optical system used to present lines of various orientations in the subject's field of view consisted essentially of a slit 3 min of arc in width and $15 \mathrm{~min}$ in length (visual angle), transilluminated by light from a tungsten halogen source and viewed at a distance of $57 \mathrm{~cm}$ through a reduction tunnel. Its luminance, measured with a United Technology meter with the photocell placed in the position of the viewing eye, was $9.6 \mathrm{~cd} / \mathrm{m}^{2}$. To permit calibrated rotations of the slit, the latter was mounted with a $360^{\circ}$ protractor on a wheel that could be rotated in the frontal plane. The protractor's markings (at $.5^{\circ}$ intervals) come to the wheel's edge to permit accurate alignment with a firmly mounted needle serving as a pointer. A plumb line was used to determine the setting of the wheel that corresponded to gravitational vertical.

\section{Procedure}

Sensitivity to the orientation of the slit was measured monocularly at $0^{\circ}$ (horizontal or $30^{\prime}$ clock position), $15^{\circ}, 30^{\circ}, 45^{\circ}$, $60^{\circ}, 75^{\circ}$, and $90^{\circ}$ (vertical or 12 o'clock position). At each of these reference orientations, the data were collected in blocks of 10 trials. Only two values of slope were presented during a given block. These two values were separated by a fixed number of degrees, and centered around a given reference orientation. Only one of the two tilts, chosen at random, was presented on a given trial. Seven different values of separation were used for each reference orientation. These were chosen on the basis of pilot data to cover the range from near indiscriminability to almost perfect resolution. For reference orientations of $30^{\circ}, 45^{\circ}$, and $60^{\circ}$, 
the separations chosen went from $1^{\circ}$ to $16^{\circ}$; for $15^{\circ}$ and $75^{\circ}$, they went from $.5^{\circ}$ to $12^{\circ}$; and for $0^{\circ}$ and $90^{\circ}$, they went from $.5^{\circ}$ to $8^{\circ}$.

The two tilts within a block were randomly presented, with the restriction that each of the two values had to appear on half of the block's trials. At the beginning of a block, the experimenter presented each of the two orientations twice, for a period of approximately $1 \mathrm{sec}$, announcing which of them was the greater (more counterclockwise) or the lesser. After these preliminary trials, the tilts were presented at random and the subject made one of the two permissible responses (greater or lesser) after each presentation, signaling with a prearranged procedure. Immediately after her response was given, the experimenter closed the shutter, told the subject the correct response, and reset the tilt for the next trial. Throughout the session, which was preceded by 2 min of dark adaptation, the subject sat in a dark room, her head fixed by means of a bite-board. The average intertrial time was approximately $5 \mathrm{sec}$ and the duration of a session was 12 min. ${ }^{2}$ Because pilot work had shown that discrimination was generally poor in the first block of trials run on an experimental day, one "warm-up" block (at $4^{\circ}$ of separation) was employed. Results of this block were not counted in the analysis of the data.

The experiment as a whole consisted of two replications per subject of the design described in the immediately following paragraph and outlined in Table 1. Each of these replications yielded the data required to obtain a measure of acuity for tilt at each of the seven reference orientations. Correlations between the measures obtained from the first and second replication were used to assess reliability of the method.

Table 1 summarizes the design for the first replication. As can be seen from the table, a Latin square was employed to counterbalance the effect of experimental session within an experimental day. Each row of the square represents 1 experimental day and shows the order in which the seven values of tilt were presented in the seven sessions constituting that day. Accordingly, in each replication of the experiment, each of the seven values of tilt $\left(0^{\circ}, 15^{\circ}, 30^{\circ}, 45^{\circ}, 60^{\circ}, 75^{\circ}\right.$, and $\left.90^{\circ}\right)$ appeared once in the first session of the day, once in the second, etc.

A second Latin square, designed to counterbalance the effect of the position of a block of trials within an experimental session, was employed at each of the seven reference orientations. To make this Latin square within a Latin square design concrete, consider the collection of data to obtain a measure of acuity for perceived tilt at $0^{\circ}$ (horizontal). Seven values of separation were chosen, ranging from $.5^{\circ}$ to $8^{\circ}$. On Day 1 , data for this value of reference orientation were collected during the first experimental session. The seven blocks within that session were run with separations of $.5^{\circ}, 1^{\circ}, 2^{\circ}, 3^{\circ}, 4^{\circ}, 6^{\circ}$, and $8^{\circ}$, in that order. On Day 2, the data for the $0^{\circ}$ reference orientation were collected during the second session. The order of the blocks within that session was $8^{\circ}, .5^{\circ}, 1^{\circ}, 2^{\circ}, 3^{\circ}, 4^{\circ}$, and $6^{\circ}$. This method of collecting data was followed for the remaining 5 days for $0^{\circ}$, as shown in the table. Similar procedures were employed with the other six values of tilt, but to avoid clutter, details are not shown in the table.

\section{RESULTS}

The raw data, which are not shown, consisted of 49 2 by 2 tables (stimulus-response matrices), one for each of the seven values of separation at each of the seven reference angles. The two column headings of an S-R matrix represented the lesser (L) and greater (G) stimulus values, each of which had been presented 70 times; the row headings ( $\ell$ and $\mathrm{g}$ ) represented the corresponding responses. The entries in the cells were the four estimated conditional probabilities: $\mathrm{P}(\ell / \mathrm{L}), \mathrm{P}(\ell / \mathrm{G}), \mathrm{P}(\mathrm{g} / \mathrm{L}), \mathrm{P}(\mathrm{g} / \mathrm{G})$. The discriminability index $\left(d^{\prime}\right)$ of signal detection theory (Green \& Swets, 1974) was calculated for each S-R matrix, assuming Gaussian distributions of equal variance and an unbiased criterion (see the immediately following section for justification of the latter assumption).

\section{Bias Analysis}

Forty-eight percent of E.M.'s responses and $49 \%$ of M.V.'s were "greater." To determine whether this represented a statistically significant bias (tendency to prefer one of the two possible responses over the other), chi square tests were performed on each of the 49 data matrices for each of the two subjects. The expected frequencies of the responses "lesser"

Table 1

Experimental Design (Separations Shown in Degrees)

\begin{tabular}{|c|c|c|c|c|c|c|c|}
\hline \multirow[b]{2}{*}{ Day } & \multicolumn{7}{|c|}{ Session } \\
\hline & 1 & 2 & 3 & 4 & 5 & 6 & 7 \\
\hline 1 & $\begin{array}{c}0 \\
(.5,1,2,3,4,6,8)\end{array}$ & 15 & 30 & 45 & 60 & 75 & 90 \\
\hline 2 & 90 & $\begin{array}{c}0 \\
(8, .5,1,2,3,4)\end{array}$ & 15 & 30 & 45 & 60 & 75 \\
\hline 3 & 75 & 90 & $\begin{array}{c}0 \\
(6,8, .5,1,2,3,4)\end{array}$ & 15 & 30 & 45 & 60 \\
\hline 4 & 60 & 75 & 90 & $\begin{array}{c}0 \\
(4,6,8, .5,1,2,3)\end{array}$ & 15 & 30 & 45 \\
\hline 5 & 45 & 60 & 75 & 90 & $\frac{0}{(3,4,6,8,5,1,2)}$ & 15 & 30 \\
\hline 6 & 30 & 45 & 60 & 75 & 90 & $\begin{array}{c}0 \\
(2,3,4,6,8, .5,1)\end{array}$ & 15 \\
\hline 7 & 15 & 30 & 45 & 60 & 75 & 90 & $\begin{array}{c}0 \\
(1,2,3,4,6,8, .5)\end{array}$ \\
\hline
\end{tabular}

Note-Main cell entries are reference orientations. Entries in parentheses are separations between tilts. Separations shown at 0 degrees only to avoid clutter. See text for further description. 
and "greater" (70 and 70 for unbiased responding) were compared with the obtained frequencies in each matrix. This analysis showed that only four values of chi square were significant ( $p$ values of .05 or less) of the 49 values calculated for subject E.M. Moreover, the sum of chi square for all of this subject's data was insignificant $(p=.12)$. Only three of M.V.'s 49 individual values of chi square were significant, and the $p$ value for the sum of chi square was .2l. These results suggest that the discriminations in the sequential recognition task, like those required in two alternative temporal or spatial forced choice procedures, tend to produce unbiased measures. ${ }^{3}$

\section{Psychometric Functions}

Because it is reasonable to assume (at least to a first approximation) that the data are unbiased, it was possible to calculate a value of $d^{\prime}$ from the single data matrix at each reference orientation rather than from an entire ROC function (see footnote 3 ). Accordingly, 49 values of $\mathrm{d}_{\mathrm{e}}^{\prime}$ ( $\mathrm{d}^{\prime}$ at equal bias) were calculated for each observer. The seven values of dé at a given reference angle were then plotted as a function of separation to yield a psychometric function. Seven of these functions were obtained,
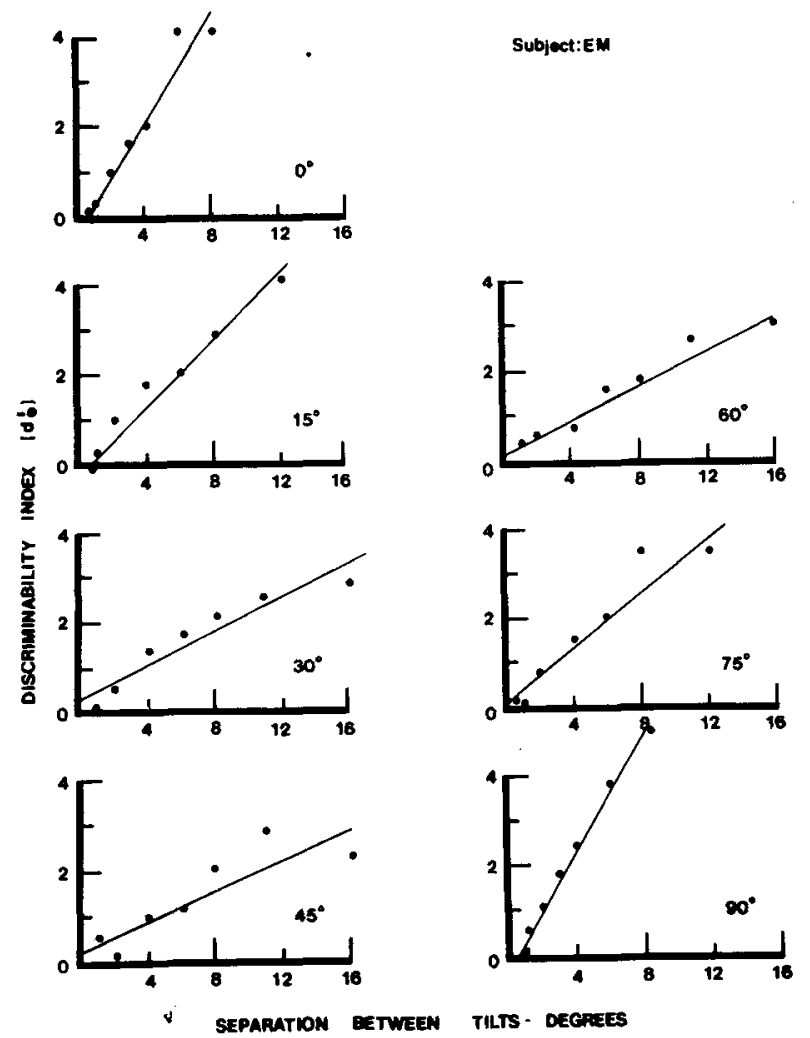

Figure 1. Psychometric functions at the seven reference orientations for subject E.M. Discriminability ( $d_{e}^{\prime}$ ) is plotted as a function of separation between the two orientations used in the discrimination. See text for further description.

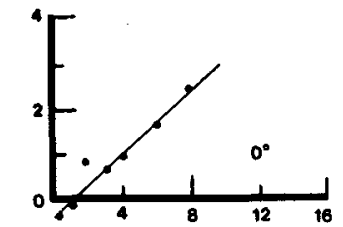

Subject: MV
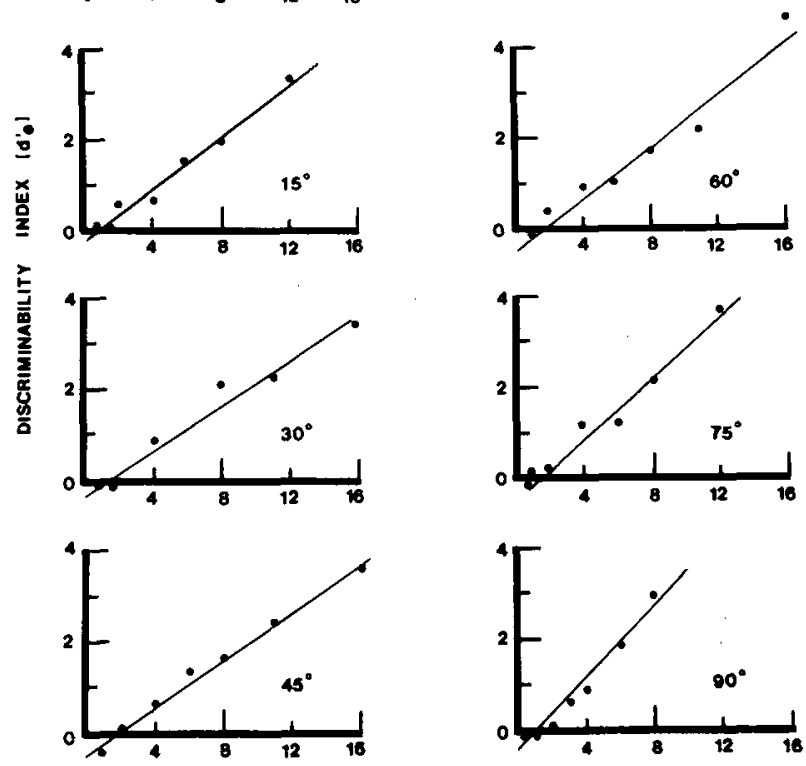

SEPARATION BETWEEN TILTS-DEGREES

Figure 2. Psychometric functions for subject M.V. Method of plotting is analagous to the one used in Figure 1.

one for each reference orientation. They are shown in Figures 1 and 2 for the combined data from the two replications of the experiment for subjects E.M. and M.V., respectively. The lines of best fit to the psychometric functions, obtained by the method of least squares, are shown along with the data points. ${ }^{4}$

\section{Measures of Sensitivity}

The psychometric functions (Figures 1 and 2) were used to derive two summary measures of sensitivity to orientation as a function of reference orientation. The first of these is shown in Figure 3 with the data for M.V. at the top and the data for E.M. at the bottom. In these plots, each curve, which we will call an isodiscriminability contour, shows the separation between angles required to produce the specified value of discriminability $\left(d_{e}^{\prime}\right)$ as a function of reference angle. As can be seen from the figure, both subjects exhibited the oblique effect; i.e., sensitivity was greater at horizontal and vertical reference angles than at the obliques. For M.V., the minima of the contours are at $30^{\circ}$; for E.M., they occur at $45^{\circ}$.

A second measure of sensitvity to orientation is shown in Figure 4. In these plots, the slope of the psychometric function is shown on the ordinate and 

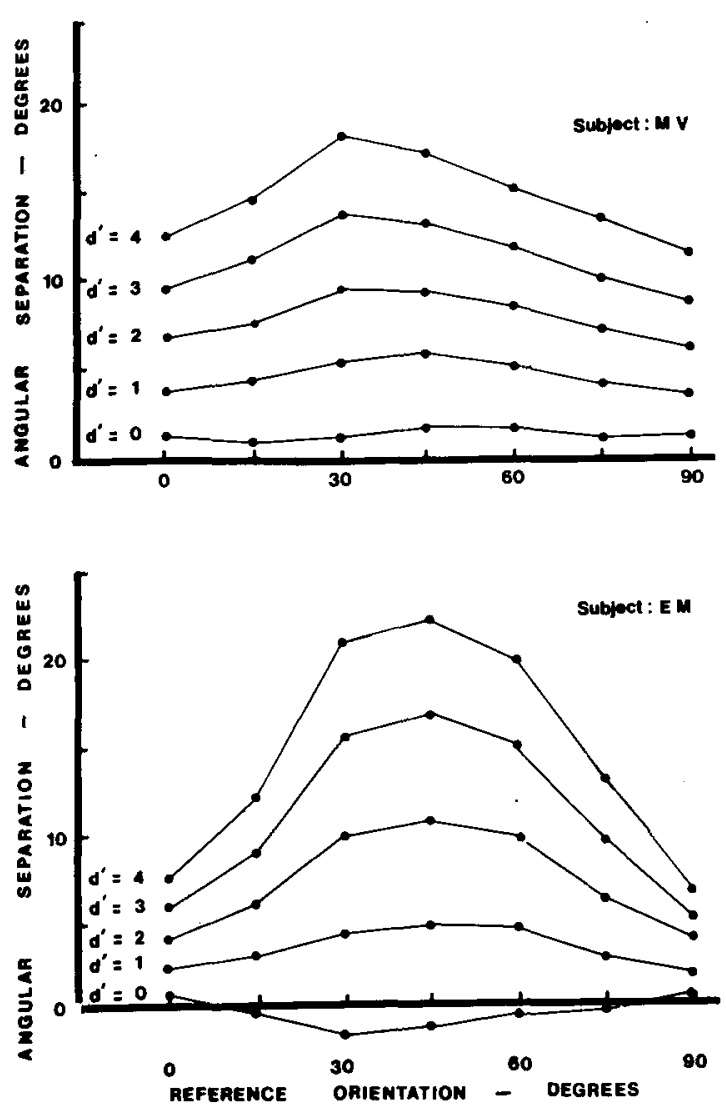

Figure 3. Isodiscriminability contours for subjects M.V. (top) and E.M. Angular separation is plotted as a function of reference orientation for five values of $\mathbf{d}_{\mathbf{e}}$. See text for further description.

reference angle on the abscissa. ${ }^{5}$ Again, the graphs clearly show the oblique effect, with maximum sensitivity at the vertical for both subjects and minimal sensitivity at $30^{\circ}$ for M.V. and $45^{\circ}$ for E.M. Strictly speaking, this method of plotting implies that there is no threshold value of separation, i.e., it implies that the intercepts of the psychometric functions are zero so that the information is contained entirely in the slopes (see Wright, 1974, for a lucid discussion of this question). As can be seen from Figure 2, this assumption does not really hold for M.V.'s data: her $\mathrm{X}$ intercepts are positive for each of the seven values of reference angle. The assumption of an intercept value of zero does seem reasonable in the case of E.M.'s data, however; four of the $\mathrm{X}$ intercepts were small negative numbers and the other three were small positive numbers. (The significance of these intercept values will be considered in the Discussion.)

\section{Reliability}

Split-half measures of the reliability of the data were obtained by correlating the slopes of the psychometric functions from the first replication with those of the second replication. This procedure yielded Pearson correlation coefficients of $.876(p=.003)$ and $.707(p=.038)$ for subjects E.M. and M.V., respectively.

\section{DISCUSSION}

This study of the oblique effect shows that reliable and sensitive measures of acuity for orientation can be obtained with sequential methods requiring only one stimulus in the visual field at any given time and presenting stimuli to the same retinal neighborhood. Thus, in effect, acuity can be measured with methods that eliminate the inadequacies of the matching techniques outlined in the introduction to this paper.

Direct comparisons with the results of previous experiments are not feasible, primarily because data from the earlier studies were processed by different statistical procedures and because stimuli employed
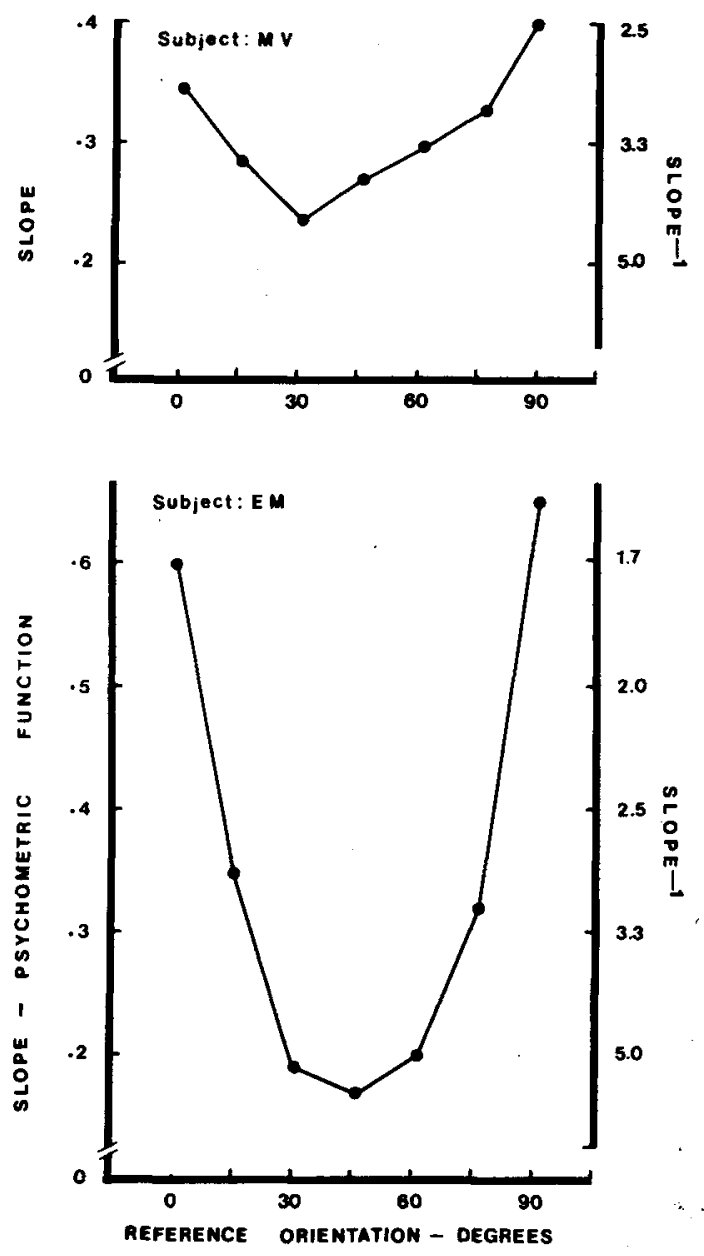

Figure 4. Slope functions for subjects M.V. (top) and E.M. Slope of the psychometric curve is plotted as a function of reference orientation. 
in those studies were presented on a luminous background, while we used luminous slits on a dark field. Other variables that are known to affect acuity, such as the time period over which data were gathered, the dimensions of the line, and exposure duration, are not comparable either. Nonetheless, it seems worth noting that orderliness of the data in this study and magnitude of the oblique effect (ratio of the most sensitive to the least sensitive points on the curve) are at least comparable to those reported in systematic studies by Andrews, who used the parallel alignment method and by Bouma and Andriessen, who used the dot alignment method (see Figure 4 in Andrews, 1967, and Figure 3 in Bouma and Andriessen, 1968). It should also be emphasized that our average intertrial time of $5 \mathrm{sec}$ (imposed by limitations of the apparatus employed) resulted in measures of acuity that clearly do not represent the most sensitive obtainable with sequential methods. Although further studies are required to determine the optimal sequencing rate, our preliminary observations leave no doubt that $5 \mathrm{sec}$ between trials is much too long.

\section{Memory in Studies of Sensory Capacity}

At first sight, it might seem that a procedure employing sequential judgments (and thus clearly tapping memory for orientation as well as sensory capacity) would be less appropriate for a parametric study of sensitivity than a procedure employing simultaneous judgments. And, indeed, though the signal recognition task and the role of memory variables have received considerable attention in the general psychophysical literature (see Sandusky, 1974, for a review), there have been, to our knowledge, no previous attempts to use such a task in "sensory psychology"-i.e., in systematic studies of intensity discrimination, hue discrimination, etc., undertaken primarily to explore sensory capacity per se rather than to study the capacity for processing information or for making psychophysical judgments.

Upon further reflection, however, it will probably seem obvious that there is no such thing as a "pure" psychophysical measure of sensory capacity, uncontaminated by other, more or less extraneous, judgmental variables. Sensory effects, criterion effects, and memory are intertwined in any discriminative task; the role of memory is simply made more obvious when a sequential method of presenting stimuli is employed. Furthermore, there is little doubt that an uncontrolled sequential factor frequently exists when subjects are required to discriminate between two simultaneously presented stimuli but are not prevented from moving their eyes from one stimulus to the other. With sequential methods, time between stimuli can be brought under experimental control. Accordingly, these techniques offer some promise for separating sensory variance from variance due to memory and other factors related to intertrial time.

\section{The Threshold Question}

In initial processing of the data, while searching for the best method for measuring sensitivity to orientation, we obtained slopes of the psychometric functions and also the $\mathrm{X}$ intercepts, expecting the latter to yield some evidence of a threshold value of separation, i.e., some minimum value below which no discrimination was possible. However, no evidence for the existence of such a threshold was found in E.M.'s psychometric functions; four of the seven X intercepts were negative, as might be expected if they were simply oscillating around a true value of 0 . Moreover, split-half reliability of the $\mathrm{X}$ intercepts for the two replications of the experiment was only .386 , again suggesting that these results essentially represented chance variation.

All of M.V.'s X intercepts were positive and could thus be construed as thresholds. This seems unlikely, however, because a low negative correlation $(r=-.489)$ was found between the first and second replications of the experiment. Moreover, even if the positive intercepts do, in fact, represent thresholds, the low correlations between replications suggests that they are independent of reference angle. Although further data will obviously be required to clarify this point, it seems reasonable to conclude that the present data yield no clear evidence for the existence of a threshold (see Swets, 1961, and Wright, 1974, for related discussions and evidence about the possible absence of a threshold in other psychophysical studies). In any event, the results of the present experiment support Wright's contention that slope of the psychometric function provides the best single measure of sensitivity (Wright, 1974).

\section{The Interpretation of Bias}

The nature of the discrimination required in the sequential recognition task suggests a priori that it, like the two alternative spatial and temporal forced choice task, would tend to produce relatively unbiased responding. Intuitively, we might say that the subject has no particular advantage in choosing "lesser" or "greater." The results corroborated this assumption; no statistically significant bias was shown, although some tendency to prefer the "lesser" response existed in both subjects. No systematic bias occurred in the direction of either the obliques, as found by Lennie (1971) with the angle matching technique, or the horizontal and vertical, as found by Bouma and Andriessen (1968) with the dot alignment technique. ${ }^{6}$

A further decrease in bias below the level observed in this study could probably be obtained by reminding subjects that the probability of both stimuli is .5 on each trial, giving them feedback at the end of 
the experimental session about the nature of their biases and encouraging them to make corrections. Such a procedure is recommended by Green and Swets for the small biases occasionally found when two-alternative, forced-choice techniques are employed (Green \& Swets, 1974, p. 108). However, given the considerable theoretical interest that has been attached to the interpretation of biases in the literature on perceived tilt (Andrews, 1967a; Bouma \& Andriessen, 1968; Emerson, et al., 1975; Lennie, 1971 ), it would probably be preferable to avoid any attempt to modify bias during the collection of data. Instead, further explorations with the sequential recognition task and with complete ROC functions should be undertaken. Such studies would permit the methods of signal detection theory to be employed in analyzing the bias question, which has been a source of considerable confusion in the tilt literature. In general, experimenters concerned with this matter have interpreted bias as a sensory effect (Andrews, 1967a; Bouma \& Andriessen, 1968; Lennie, 1971). However, the direction of the bias (toward or away from the main spatial axes) is a function of the method chosen for measuring perceived tilt (Emerson et al., 1975). This fact suggests (but certainly does not prove) that response biases, not sensory biases, could be responsible for the phenomenon.

\section{Choice of Stimuli in Measuring Sensitivity}

An advantageous feature of the present method that could easily be extended to other studies derives from the fact that the angular separations for which discriminability was measured were all centered around the reference orientation. In assessing sensitivity at $60^{\circ}$, for example, we measured the ability to discriminate between $59.5^{\circ}$ and $60.5^{\circ} ; 59^{\circ}$ and $61^{\circ}$; $58^{\circ}$ and $62^{\circ} ; 57^{\circ}$ and $63^{\circ} ; 56^{\circ}$ and $64^{\circ} ; 54.5^{\circ}$ and $65.5^{\circ}$; and $52^{\circ}$ and $68^{\circ}$. At the largest value of separation $\left(16^{\circ}\right)$, the orientations of the lines employed in the discriminative task differed from the reference orientation of $60^{\circ}$ by only $8^{\circ}$. This procedure should be compared to the conventional methods with simultaneous pair comparisons in which it is customary to employ one standard reference stimulus and several comparison stimuli separated from the standard by varying amounts. If the latter methods had been followed in the present study, we would have employed $60^{\circ}$ and $61^{\circ}, 60^{\circ}$ and $62^{\circ} \ldots$, $60^{\circ}$ and $76^{\circ}$ to achieve the separations of $1^{\circ}, 2^{\circ}$, $4^{\circ}, 8^{\circ}, 11^{\circ}$, and $16^{\circ}$ used in our study. At the largest value of separation, the $76^{\circ}$ comparison stimulus would have been $16^{\circ}$ away from the reference orientation, twice the distance required with the "straddling" method of the present study. The importance of minimizing distance from the reference orientation arises from the fact that sensitivity changes with reference orientation. Because sensitivity changes, however, the ability to discriminate at the reference orientation should be assessed with stimuli as close as possible to that orientation. ${ }^{7}$

Needless to say, the straddling feature of the method employed in this study does not depend on use of the sequential recognition task; it could easily be incorporated in studies employing simultaneous pair comparisons as the raw measure of discriminative capacity. Nor are the advantages of the straddling procedures peculiar to studies of the oblique effect. Considerations similar to those outlined in the preceding paragraph obviously also apply in studies of hue discrimination or in studies concerned with Weber's law. Indeed, they apply in any psychophysical study concerned with the measurement of sensitivity as a function of some stimulus variable.

\section{REFERENCE NOTE}

1. Alluisi, E. (Ed.). Lineal inclination in encoding information symbolically on cathode ray tubes and similar displays. USAF ASD Technical Report No. 61-741, December 1961.

\section{REFERENCES}

ANDREws, D. Perception of contours in the central fovea. Nature, 1965, 205, 1218-1220.

ANDrews, D. Perception of contour orientation in the central fovea. I. Short lines. Vision Research, 1967, 7, 975-979. (a)

ANDREwS, D. Perception of contour orientation in the central fovea. II. Spatial integration. Vision Research, 1967, 7, 999-1013. (b)

Appelle, S. Perception and discrimination as a function of stimulus orientation: The "oblique effect" in man and animals. Psychological Bulletin, 1972, 78, 266-278.

Blakemore, C., Carpenter, R., \& Georgeson, M. Lateral inhibition between orientation detectors in the human visual system. Nature, 1970, 228, 37-39.

Bouma, H., \& ANDriessen, J. J. Perceived orientation of isolated line segments. Vision Research, 1968, 8, 493-507.

Bouma, H., \& ANDriessen, J. Induced changes in the perceived orientation of isolated line segments. Vision Research, 1970, 10, 333-349.

Campbell, F., \& Maffei, L. The tilt aftereffect: A fresh look. Vision Research, 1971, 11, 833-840.

CARpenter, R., \& Blakemore, C. Interactions between orientations in human vision. Experimental Brain Research, 1973, 18, 287-303.

Emerson, P., Wenderoth, P., Curthoys, I., \& Edmonds, I. Measuring perceived orientation. Vision Research, 1975, ,15, 1031-1033.

Finney, D. Probit analysis. Cambridge: Cambridge University Press, 1971.

FISHER, G. An experimental study of angular subtension. Quarterly Journal of Experimental Psychology, 1969, 21, 356-366.

FISHER, G. But if they either are not what they seem or seem what they are not, then how can the perceptual distortions be measured? Perception, 1973, 2, 165-166.

Fisher, G. An experimental study of linear inclination. Quarterly Journal of Experimental Psychology, 1974, 18, 52-62.

Georgeson, M. Psychophysical illusions of orientation and spatial frequency. Perception, 1976, 5, 99-111.

GREEN, D., \& SwETs, J. Signal detection theory and psychophysics. Huntington: Krieger, 1974. 
Gunforo, J. Psychometric methods. New York: McGraw-Hill, 1954.

JASTROW, J. On the judgment of angles and positions of lines. American Journal of Psychology, 1893, 5, 214-248.

KEENE, G. The effect of response codes on the accuracy of making absolute judgments of lineal inclinations. Journal of General Psychology, 1963, 69, 37-50.

LENNIE, P. Distortions of perceived orientation. Nature, New Biology, 1971, 233, 155-156.

Matin, E. Light adaptation and the dynamics of induced tilt. Vision Research, 1974, 14, 255-265.

Mitchell, D., \& MUIR, D. Does the tilt aftereffect occur in the oblique meridian? Vision Research, 1976, 16, 609-613.

MuIR, D., \& Over, R. Tilt aftereffects in central and peripheral vision. Journal of Experimental Psychology, 1970, 85, 165-170.

O'TOOle, B., \& Wenderoth, P. The tilt illusion: Repuision and attraction effects in the oblique meridian. Vision Research, $1977,17,367-374$.

Over, R., Broerse, J., \& Crassini, B. Orientation illusion and masking in central and peripheral vision. Joumal of Experimental Psychology, 1972, 96, 25-31.

Oyama, T. Determinants of Zöllner illusion. Psychological Research, 1975, 37, 261-280.

Rochlin, A. The effect of tilt on the visual perception of parallelness. American Journal of Psychology, 1955, 68, 223-236.

SAlomon, A. Visual field factors in the perception of direction. American Journal of Psychology, 1947, 60, 68-88.

Sandusxy, A. Memory processes and judgment. In C. Carterette \& M. Friedman (Eds.), Handbook of perception (Vol. II) Psychophysical judgment and measurement. New York: Academic Press, 1974.

Sмiтн, S. Angular estimation. Journal of Applied Psychology, 1962, 46. 240-246.

SwETs, J. Is there a sensory threshold? Science, 1961, 134, 168-177.

Thomas, J., \& SHImamura, K. Inhibitory interactions between visual pathways tuned to different orientations. Vision Research, $1975,15,1373-1380$.

Tolnurst, D., \& Thompson, P. Orientation illusions and aftereffects. Vision Research, 1975, 15, 967-972.

Wallace, G. The effect of contrast on the Zöllner illusion. Vision Research, 1975, 15, 963-966.

Wallace, G., \& Crampin, D. The effect of background density on the Zöllner illusion. Vision Research, 1969, 9, 167-177.

Wenderoth, P., Beh, H., \& White, D. Alignment errors to both ends of acute- and obtuse-angle arms. Perception \& Psychophysics, 1978, 23, 475-482.

WENDEROTh, P., \& CuRthoys, I. On the nonadditivity of visual tilt illusions. Quarterly Journal of Experimental Psychology, 1974, 26, 549-555.

Wrugrr, A. Psychometric and psychophysical theory within a framework of response bias. Psychological Review, 1974, 81, 322-347.

WrIGHT, A. Construction of equal hue discriminability scales for the pigeon. Journal of the Experimental Analysis of Behavior, $1978,29,261-266$.

\section{NOTES}

1. The oblique effect is a superiority in performing a variety of visual discriminations when stimuli are horizontal or vertical rather than oblique. One manifestation of this superiority is found in studies of acuity for orientation. See Appelle, 1972, for a review and an extensive list of references.

2. Short blocks of trials and short experimental sessions are desirable in obtaining measurements of acuity, because results can be seriously affected by any lapse of the subject's attentiveness. We also wish to emphasize the desirability of explicit feedback from the experimenter.

3. Forced choice techniques have been popular in sensory psychology because they tend to produce unbiased responding and thus permit satisfactory measurement of sensitivity without the inconvenience of obtaining an ROC function. See Green and Swets, 1974, p. 408, for a discussion.

4. Inspection of Figures 1 and 2 suggests that linear functions provide reasonably good fits to the psychometric curves. However, we will argue elsewhere that the "true" psychometric functions for sensitivity to orientation are not linear (Matin and Drivas, in preparation).

5. Slope of the psychometric function (ratio of change in discriminability to change in separation between stimuli) varies directly with discriminability. Note, however, that its reciprocal (slope ${ }^{-1}$ ) is the measure of choice for expressing relative sensitivity (see Wright, 1978, p. 263). The former is plotted in linear steps on the left axis of Figure 4. The corresponding values of slope ${ }^{-1}$ are shown on the right axis. These reciprocal values are directly proportional to the standard deviations of the normal ogives.

6. To avoid confusion, it is important to note that the word "bias" is used in different but related ways in the literatures on perceived tilt and on signal detection theory. In the former, bias refers to a mean perceived tilt that is different from the physical tilt. Assume, for example, that the mean perceived tilt is $78^{\circ}$ when measurements are made with a line physically slanted at $75^{\circ}$. This finding would be referred to as a bias toward the vertical. The technique employed in the present study is designed only to obtain measures of sensitivity (analagous to the standard deviations of the distribution of responses in other studies of perceived tilt). However, a tendency to prefer "greater" to "lesser" at $75^{\circ}$ (i.e., a bias toward "greater") would presumably manifest itself as a mean perceived tilt in the direction of $90^{\circ}$ if the conventional methods of measuring perceived orientation were being employed.

7. For the purposes of this study, which were primarily methodological, we were naturally interested in exploring the entire uncertainty region. Accordingly, we used a relatively large number of separations (seven), spread over a large range. For studies that are primarily substantive, however, the use of a large number of separations is both inefficient and unnecessary. Indeed, given that sensitivity changes with orientation, it seems clear that stimulus values near the extremes of the psychometric function should be avoided; they will necessarily be located at a considerable distance from the reference orientation, even with the straddling technique employed in this study. These distant points are those, moreover, that are given low weights for entirely different reasons when normal ogives are fit by the method of least squares and by the probit method. (See Guilford, 1954, and Finney, 1971, for discussions of the Müller-Urban weights with the least squares and probit techniques, respectively.)

(Received for publication October 3, 1978; revision accepted December 12,1978 .) 